\title{
Neuromodulation for low-anterior resection syndrome
}

\author{
Niamh McCawley ${ }^{1,2}$ \& P Ronan O'Connell*,1,2 \\ ${ }^{1}$ School of Medicine \& Medical Sciences, University College Dublin, Belfield, Dublin 4 D04 T6F4, Ireland \\ ${ }^{2}$ Surgical Professorial Unit \& Department of Colorectal Surgery, St Vincent's University Hospital, Elm Park, Nutley Lane, Dublin D04 \\ T6F4, Ireland \\ * Author for correspondence: Tel.: +35 31221 4000; ronan.oconnell@ucd.ie
}

\section{"Anterior resection has many benefits for the patient, not least avoiding the prospect of a permanent colostomy, but comes at a high functional price."}

First draft submitted: 30 August 2017; Accepted for publication: 26 September 2017; Published online: 7 December 2017

Keywords: fecal incontinence • low anterior resection syndrome • rectal cancer

Surgery remains the definitive curative treatment for rectal cancer. The surgical approach involves complex pelvic dissection and mandates a negative distal resection margin. Contemporary techniques and multimodal management, with the advent of circular stapling devices and the use of neoadjuvant chemoradiotherapy, have translated into a higher rate of a sphincter salvage approach with, for example, low-anterior resection. Indeed, there has been an evolution in modern surgical practice to achieve a diminishingly negative distal resection margin with the feasibility of ultra low resection and cancer-free survival succinctly proven [1].

Anterior resection has many benefits for the patient, not least avoiding the prospect of a permanent colostomy, but comes at a high functional price. The functional disturbances experienced by patients following low anterior resection can be extremely debilitating and may have a substantial negative impact on quality of life following a resection. The symptoms range from frequent episodes of flatal and fecal incontinence, stool clustering, increased stool frequency and urgency to contrasting symptoms of constipation and obstructed defecation. This constellation of symptoms has been termed low-anterior resection syndrome (LARS). Despite the regularity of experiencing these symptoms following surgery and their associated distress, there remains no specific unifying treatment modality to address this common problem. Strategies have focused upon attaining symptom relief with medication, pelvic floor rehabilitative physiotherapy and biofeedback, trans-anal rectal irrigation, the use of anal plugs and more recently sacral neuromodulation.

LARS is common, with $25-80 \%$ of patients postsphincter-saving anterior resection experiencing one or more symptom [2-4]. Previously, symptoms were thought to be transient following resectional-pelvic surgery, resolving in the main, by 12 months postoperatively [5]. Longer term cohort studies have identified symptoms remaining at 15 years after surgery [3], suggesting enduring changes rather than short term irritability of the neorectum.

\section{Pathophysiology of LARS}

The cause of the symptoms is thought to be multifactorial, with studies implicating anal sphincter damage, postoperative colonic dysmotility and reduced reservoir capacity and compliance of the neo-rectum. Surgical factors such as anastomotic technique and the varying options in construction of a new reservoir (colonic J-pouch, side to end anastomosis, transverse coloplasty), add to the complexity in identifying a single theory with which to attribute these gastrointestinal symptoms.

Structural and/or neurological damage to the anal sphincter complex at anterior resection has been implicated in developing passive incontinence and decreased urge time, with consequent urge incontinence. Studies have identified reduced anal canal resting pressures following anterior resection which fails to ameliorate over time [6,7] and also diminished maximum anal canal squeeze pressures [8] at manometric assessment of the postoperative anal sphincter. 
Many studies have observed the link between treatment modality and the development of LARS, with the use of neoadjuvant radiotherapy and now seldom used adjuvant radiotherapy as leading contributors in its development. Data from the Dutch Total Mesorectal Excision (TME) trial observed increased rates of fecal incontinence in patients who underwent short course preoperative radiotherapy, compared with patients who underwent surgery alone [9]. Similar results have been observed following the use of long course radio-chemotherapy demonstrating a deterioration in bowel function [4,10]. These studies identify a causal relationship between radiotherapy and the subsequent development of LARS, however modern targeted radiotherapy regimes and refined laparoscopic low-anterior resection, employing the now ubiquitous TME technique, render some of these older studies difficult to interpret in today's modern rectal cancer practice.

In addition, the level of the anastomosis, with the lower level of anastomosis, and anastomotic complications, such as leak, have been implicated as risk factors for the development of LARS. More recently studies have identified an increased likelihood of subsequent development of LARS with a TME to the level of the pelvic floor, in comparison to a partial mesorectal excision.

\section{Diagnosis \& treatment of LARS}

The diagnosis of LARS, in addition to careful history and full physical examination, is augmented with the addition of validated questionnaires [11,12], to determine the severity of LARS symptoms. The most used scoring system, LARS score, stratifies patients into minor and major LARS, with a score $>30$ deemed major LARS and indicative of a profound compromise in quality of life. Major LARS usually requires multimodal treatments, including pelvic floor muscle training, biofeedback and trans-anal irrigation therapy. In most treatment algorithms, following initiation of treatments, LARS scores are revaluated at an appropriate interval, usually a year; and those who do not improve may be offered a trial of sacral neuromodulation.

\section{Sacral neuromodulation}

Sacral neuromodulation involves the low voltage stimulation of the sacral nerve roots and is commonly termed sacral nerve stimulation (SNS). The procedure involves the positioning of an electrode into one of the sacral foramina, usually S3, to induce low-grade electrical stimulation to the sacral nerve. Following a 2 -week trial period with a temporary electrode, termed percutaneous nerve evaluation, patients are assessed for response, with responders having the option to have a permanent electrode implanted subcutaneously.

The mechanism of action of SNS is not fully elucidated. Initial studies suggested a direct action on the anal sphincter mediated by peripheral motor stimulation resulting in an increase in resting and squeeze pressures; however more recent evidence points to an action at a pelvic afferent or central level [13]. Further studies examining the mechanism of action of SNS have demonstrated a decrease in antegrade while increasing retrograde colonic motor activities [14]. Diminished postprandial variations in rectal motility have also been demonstrated in a separate study [15]. These mechanisms may have particular resonance for patients with symptoms of LARS.

A systematic review of sacral neuromodulation for LARS, published in 2015, reviewed seven pertinent papers, with only 43 patients evaluated, with mean follow-up of 15 months. 94.1\% of those fitted with a permanent implant, following a successful percutaneous nerve evaluation, experienced an improvement of symptoms, with an intention to treat success rate of $74.4 \%[16]$.

Another review of SNS for fecal incontinence following rectal cancer and radiotherapy highlighted the heterogeneity in operations performed, doses of radiotherapy received and the wide variation in patient symptoms as barriers to draw meaningful conclusions, however, they did note that implantation of SNS improved symptoms and quality of life in patients [17].

A recently published study, again with small numbers, 11 patients implanted, concluded that SNS is useful in all symptoms of LARS, such as fragmentation and urgency, and not just fecal incontinence [18].

Percutaneous tibial nerve stimulation is an alternative minimally invasive technique for neurostimulation, utilizing the posterior tibial nerve at the ankle, that had gained popularity for the treatment of fecal incontinence [19,20]. It has not been formally investigated as a treatment modality for LARS. However, the publication of the randomized controlled trial (CONtrol of Fecal Incontinence using Distal NeuromodulaTion [CONFIDeNT]) [21], demonstrating no benefit of percutaneous tibial nerve stimulation over sham electrical stimulation may prove the death knell for this therapy. 
Many opponents of sacral neuromodulation have highlighted the high cost of the procedure when compared with conservative measures such as biofeedback and conservative medical management, however its cost-effectiveness is evident when offset by the quality-adjusted life years gained for patients [22].

\section{Conclusion}

LARS is common following anterior resection. It has debilitating sequelae for patients, made all the starker when taken in the context of patients surviving a potentially life-threatening condition, only to face incapacitating consequences of cure.

The balance of achieving meticulous oncological clearance and preserving anal sphincter function is challenging for surgeons caring for rectal cancer patients, and in particular, those with low rectal cancer. Many have questioned the benefit for patients in performing ultra-low anastomoses. Indeed, recent programs advocating for improved outcomes of low rectal cancer, such as The English National Low Rectal Cancer Development Program (LOREC) and the English National Cancer Action Team to improve the outcome of low rectal cancer in England, have focused on abdominoperineal excision. For restorative procedures, the potentially preventative measures of careful nervesparing surgery, adherence to stringent pelvic irradiation protocols is essential, as is tempering patients' expectations to realistic outcomes. As quality of life impairment is common following a restorative anterior resection, patients with rectal cancer should be adequately counseled on the potential bowel-related consequences [23]. Indeed, a recent international collaboration of UK and Danish surgeons has sought to develop a tool to predict bowel dysfunction severity prior to anterior resection. The Pre-operative LARS (POLARS) score purports to help patients understand their risk of bowel dysfunction and to preoperatively highlight patients who may require additional postoperative support [24].

Sacral neuromodulation offers the prospect of improvement in symptoms and consequently enhanced quality of life. However, it is imperative to note that there is a paucity of evidence examining the effect of SNS on LARS with only retrospective studies with small numbers of patients available for scrutiny, heterogeneity in the variables studied and the inherent difficulty in comparative statistical analysis.

Prospective studies with long term follow-up and widespread use of validated questionnaires are required to allow for meaningful conclusions and comparisons to be drawn in respect of the most appropriate treatment modalities for LARS, and an integrated postoperative functional follow-up, with access to rehabilitative physiotherapy and sacral neuromodulation, if required, should complement the established oncologic patient journey.

\section{Financial \& competing interests disclosure}

The authors have no relevant affiliations or financial involvement with any organization or entity with a financial interest in or financial conflict with the subject matter or materials discussed in the manuscript. This includes employment, consultancies, honoraria, stock ownership or options, expert testimony, grants or patents received or pending, or royalties.

No writing assistance was utilized in the production of this manuscript.

\section{References}

1. Rullier E, Denost Q, Vendrely V, Rullier A, Laurent C. Low rectal cancer: classification and standardization of surgery. Dis. Colon Rectum 56(5), 560-567 (2013).

2. Ekkarat P, Boonpipattanapong T, Tantiphlachiva K, Sangkhathat S. Factors determining low anterior resection syndrome after rectal cancer resection: a study in Thai patients. Asian J. Surg. 39(4), 225-231 (2016).

3. Chen TY, Wiltink LM, Nout RA et al. Bowel function 14 years after preoperative short-course radiotherapy and total mesorectal excision for rectal cancer: report of a multicenter randomized trial. Clin. Colorectal Cancer 14(2), 106-114 (2015).

4. Bregendahl S, Emmertsen KJ, Lous J, Laurberg S. Bowel dysfunction after low anterior resection with and without neoadjuvant therapy for rectal cancer: a population-based cross-sectional study. Colorectal Dis. 15(9), 1130-1139 (2013).

5. Pedersen IK, Christiansen J, Hint K, Jensen P, Olsen J, Mortensen PE. Anorectal function after low anterior resection for carcinoma. Ann. Surg. 204(2), 133-135 (1986).

6. Williamson ME, Lewis WG, Finan PJ, Miller AS, Holdsworth PJ, Johnston D. Recovery of physiologic and clinical function after low anterior resection of the rectum for carcinoma: myth or reality? Dis. Colon Rectum 38(4), 411-418 (1995).

7. Pucciarelli S, Del Bianco P, Efficace F et al. Health-related quality of life, faecal continence and bowel function in rectal cancer patients after chemoradiotherapy followed by radical surgery. Support Care Cancer 18(5), 601-608 (2010).

8. Horgan PG, O'Connell PR, Shinkwin CA, Kirwan WO. Effect of anterior resection on anal sphincter function. Br. J. Surg. 1989. 76(8), 783-786 (1989). 
9. Lange MM, den Dulk M, Bossema ER et al. Cooperative clinical investigators of the Dutch Total Mesorectal Excision trial. Risk factors for faecal incontinence after rectal cancer treatment. Br. J. Surg. 94(10), 1278-1284 (2007).

10. Qin Q, Huang B, Cao W et al. Bowel dysfunction after low anterior resection with neoadjuvant chemoradiotherapy or chemotherapy alone for rectal cancer: a cross-sectional study from China. Dis. Colon Rectum 60(7), 697-705 (2017).

11. Emmertsen KJ, Laurberg S. Low anterior resection syndrome score: development and validation of a symptom-based scoring system for bowel dysfunction after low anterior resection for rectal cancer. Ann. Surg. 255(5), 922-928 (2012).

12. Temple LK, Bacik J, Savatta SG et al. The development of a validated instrument to evaluate bowel function after sphincter-preserving surgery for rectal cancer. Dis. Colon Rectum 48(7), 1353-1365 (2005).

13. Carrington EV, Evers J, Grossi U et al. A systematic review of sacral nerve stimulation mechanisms in the treatment of fecal incontinence and constipation. Neurogastroenterol. Motil. 26(9), 1222-1237 (2014).

14. Michelsen HB, Christensen P, Krogh K et al. Sacral nerve stimulation for faecal incontinence alters colorectal transport. Br. J. Surg. 95(6), 779-784 (2008).

15. Michelsen HB, Worsøe J, Krogh K et al. Rectal motility after sacral nerve stimulation for faecal incontinence. Neurogastroenterol. Motil. 22(1), 36-41, e6 (2010).

16. Ramage L, Qiu S, Kontovounisios C, Tekkis P, Rasheed S, Tan E. A systematic review of sacral nerve stimulation for low anterior resection syndrome. Colorectal Dis. 17(9), 762-771 (2015).

17. Thomas GP, Bradshaw E, Vaizey CJ. A review of sacral nerve stimulation for faecal incontinence following rectal surgery and radiotherapy. Colorectal Dis. 17(11), 939-942 (2015).

18. D’Hondt M, Nuytens F, Kinget L, Decaestecker M, Borgers B, Parmentier I. Sacral neurostimulation for low anterior resection syndrome after radical resection for rectal cancer: evaluation of treatment with the LARS score. Tech. Coloproctol. 21(4), 301-307 (2017).

19. Boyle DJ, Prosser K, Allison ME, Williams NS, Chan CL. Percutaneous tibial nerve stimulation for the treatment of urge fecal incontinence. Dis. Colon Rectum 53(4), 432-437 (2010).

20. Govaert B, Pares D, Delgado-Aros S, La Torre F, Van Gemert WG, Baeten CG. A prospective multicentre study to investigate percutaneous tibial nerve stimulation for the treatment of faecal incontinence. Colorectal Dis. 12(12), 1236-1241 (2010).

21. Knowles $\mathrm{CH}$, Horrocks EJ, Bremner SA et al. Percutaneous tibial nerve stimulation versus sham electrical stimulation for the treatment of faecal incontinence in adults (CONFIDeNT): a double-blind, multicentre, pragmatic, parallel-group, randomised controlled trial. Lancet 386(10004), 1640-1648 (2015).

22. Chiarioni G, Palsson OS, Asteria CR, Whitehead WE. Neuromodulation for fecal incontinence: an effective surgical intervention. World J. Gastroenterol. 19(41), 7048-7054 (2013).

23. Battersby NJ, Juul T, Christensen P et al. Predicting the risk of bowel-related quality-of-life impairment after restorative resection for rectal cancer: a multicenter cross-sectional study. Dis. Colon Rectum 59(4), 270-280 (2016).

24. Battersby NJ, Bouliotis G, Emmertsen KJ et al. Development and external validation of a nomogram and online tool to predict bowel dysfunction following restorative rectal cancer resection: the POLARS score. Gut doi:10.1136/gutjnl-2016-312695 (2017) (Epub ahead of print). 